\title{
The effects of the binocular disparity differences between targets and maskers on visual search
}

\author{
Ya-Yue Gao ${ }^{1} \cdot$ Bruce Schneider $^{3} \cdot{\operatorname{Liang~} \mathrm{Li}^{1,2}}^{1,2}$
}

Published online: 9 December 2016

(C) The Psychonomic Society, Inc. 2016

\begin{abstract}
A visual search for targets is facilitated when the target objects are on a different depth plane than other masking objects cluttering the scene. The ability of observers to determine whether one of four letters presented stereoscopically at four symmetrically located positions on the fixation plane differed from the other three was assessed when the target letters were masked by other randomly positioned and oriented letters appearing on the same depth plane as the target letters, or in front, or behind it. Three additional control maskers, derived from the letter maskers, were also presented on the same three depth planes: (1) random-phase maskers (same spectral amplitude composition as the letter masker but with the phase spectrum randomized); (2) random-pixel maskers (the locations of the letter maskers' pixel amplitudes were randomized); (3) letter-fragment maskers (the same letters as in the letter masker but broken up into fragments). Performance improved with target duration when the targetletter plane was in front of the letter-masker plane, but not when the target letters were on the same plane as the masker, or behind it. A comparison of the results for the four different kinds of maskers indicated that maskers consisting of recognizable objects (letters or letter fragments) interfere more with search and comparison judgments than do visual noise
\end{abstract}

Liang Li

liangli@pku.edu.cn

1 School of Psychological and Cognitive Sciences, Beijing Key Laboratory of Behavior and Mental Health, Key Laboratory on Machine Perception (Ministry of Education), Peking University, Beijing, China 100080

2 Beijing Institute for Brain Disorder, Capital Medical University, Beijing, China

3 Department of Psychology, University of Toronto, Mississauga, Ontario, Canada maskers having the same spatial frequency profile and contrast. In addition, performance was poorer for letter maskers than for letter-masker fragments, suggesting that the letter maskers interfered more with performance than the letterfragment maskers because of the lexical activity they elicit.

Keywords Visual search · Visual masking · Informational masking $\cdot$ Energetic masking $\cdot$ Binocular unmasking · Camouflage breaking $\cdot$ Stereopsis $\cdot$ Perceptual separation . Attention

\section{Introduction}

\section{Stereopsis and binocular unmasking}

Visual masking refers to a reduction in the visibility of the target due to the presentation of masking objects nearby in space and/or time (Enns \& Di Lollo, 2000). Hence, we would expect the presence of masking objects to interfere more with the search for visual targets the closer the masker objects are to the targets in depth. Indeed, the search time for a target object increases the closer in depth distracting flanking objects are to the target object (de la Rosa, Moraglia, \& Schneider, 2008). Thus, stereopsis is critical not only for localizing objects in depth (Howard \& Rogers, 1995, 2002, 2012) but also for unmasking the target (e.g., Heesy, 2009; Henning \& Hertz, 1973; Schneider, Moraglia, \& Jepson, 1989). Specifically, when a target is presented stereoscopically against a masking background, a difference in binocular disparity between the target and masker significantly improves the detection of the target(Henning \& Hertz, 1973; Moraglia \& Schneider, 1990, 1992; Otto, Bach, \& Kommerell, 2010; Speranza, Moraglia, $\&$ Schneider,1995, 2001; Wardle et al. 2010). The reduction of masking due to stereoscopic disparity has been called 
binocular unmasking and the unmasking of simple targets such as Gabor stimulus presented in a Gaussian noise background has been shown to be dependent on the spectral characteristics of the summated images on the two retinas (Moraglia \& Schneider, 1992; Schneider et al., 1989).

An interesting aspect of the binocular unmasking of a Gabor target in a noise background is that the degree of unmasking does not appear to depend on whether the target is perceived to be in front of or behind the plane of the noise masker, or even on whether the induced disparity difference is along the horizontal or vertical plane, or some combination of the two. Rather it appears to depend primarily on the spectral profile of the summated target relative to that of the summated masker. If the spectral profile of the left- and right-eye summated images of the target falls in a trough in the spectral profile of the left- and right-eye summated images of the masker, binocular unmasking occurs irrespective of the type of binocular disparity difference between the masker and target. Hence this type of binocular unmasking appears to be independent of the type of disparity (crossed versus uncrossed) induced in the masker.

\section{Crossed disparity versus uncrossed disparity}

Relative to a fixation plane with zero disparity, if the binocular disparity of a stereoscopically presented object is of negative value (called "crossed"), the object would be perceived to be nearer to the observer than an object on the fixation plane; if the binocular disparity is of positive value (called "uncrossed"), the object would be perceived to be farther away from the observer than an object on the fixation plane. It has become evident that binocular disparity information in stereopsis is encoded by different mechanisms that are selective for crossed and uncrossed disparities, respectively (e.g., Richards, 1970, 1971; for a review see Mustillo, 1985).

There are reasons to believe the ability of an observer to detect, locate, and identify a target could depend on whether or not the masking stimuli were presented on the same plane as that of the target or with either uncrossed (appearing on a plane behind the target) or crossed (appearing on a plane in front of the target) disparity. As mentioned before, it has been reported that the detection of a simple target (such as a Gabor stimulus) presented at the point of fixation is independent of whether a visual noise masker is presented in either crossed or uncrossed disparity relative to the target plane (e.g., Moraglia and Schneider; 1990; Uttal, Fitzgerald, \& Eskin,1975; Wardle et al., 2010). However, there are a number of studies demonstrating that the ability to locate or identify an object (as opposed to detecting a Gabor pattern) does depend on the type of disparity between target and masking planes (e.g., Fox \& Patterson, 1981; Lehmkuhle \& Fox, 1980; Nakayama \& Silverman, 1986; O’Toole \& Walker, 1997; Manning, Finlay, Neill, \& Frost, 1987; Patterson et al., 1995). The present study is designed to help identify the conditions in which the direction of the disparity makes a difference in tasks involving a search component.

\section{Object formation and the perception of a depth plane}

When the binocular disparity of the target is different from that of any masking objects, the target and the masking objects are perceived as being located at different depths. When simple targets (such as Gabor stimuli) are presented at the point of fixation, the type of disparity of a Gaussian noise masker (crossed versus uncrossed) appears to be irrelevant (Moraglia \& Schneider, 1990) with respect to the detectability of the Gabor target. However, when the task involves determining the location and/or identification of a target object (e.g., lines, squares, circles), presented in the presence of a masker plane containing similar or different objects, the sign and degree of disparity of the masking plane might make a difference. To the extent that object formation occurs subsequent to a spatial frequency decomposition of the stimulus, it is possible that whether or not the masker plane is in front of or behind the target will make a difference when identifiable objects appear on either the focal plane or on the masker plane. When the target is a Gabor stimulus on the focal plane, and the masker is a Gaussian noise plane that appears behind the target plane, the Gabor appears to float in front of but not obscure the Gaussian masker. When the target appears behind the masker plane, the target appears to float behind a transparent Gaussian masker (Schneider et al., 1989; Moraglia \& Schneider, 1990). However, when the masker plane as well as the target plane consists of a collection of identifiable visual objects (e.g., the letters of the alphabet), there are reasons to believe that objects that are closer to the observer would have a higher salience than those that are further away (the behavior urgency hypothesis, Franconeri and Simons, 2003). For instance, if one is walking through a cluttered field, or through a room filled with children's toys, objects that are close to observers will require one's immediate attention. A tendency for nearby objects to more easily capture attention than more distant ones, would lead us to expect search and comparison operations to proceed more rapidly when the target objects are in front of the masking objects, than when they are behind them.

\section{Object identification in a cluttered two-dimensional field}

In viewing natural scenes, it is not only important to detect and locate objects in the visual field, it is also often necessary to determine their identity. The identification of a searched-for object in a visual field has been shown to depend on how cluttered the field is with other objects (e.g., Pelli \& Tillman, 2008). The more cluttered the visual field, the more difficult it is to locate and identify a searched-for object. Moreover, the ability to identify a searched-for object appears to depend on 
the similarity of the target object to the other objects in the visual field. Searching for the letter "A" in a field of letters is much faster when the target letter is of a different color than the other letters in the field. In such instances, the target letter appears to "pop out" from the background (e.g., Treisman \& Glade, 1980).

Interestingly, the effect of the similarity of the target object to that of distractors, appears to depend on whether one is searching for the target among distractors or identifying a target whose location is known. Rabaglia and Schneider (2016) found that young adults could more readily identify a single English letter, presented at a known location (the fixation point), when it was surrounded by a field of other randomly oriented letters, than they could when the surrounding letters were fragmented, suggesting that the more similar the surrounding objects in the visual field to that of the target, the easier it is to identify the target letter. Hence the effect of distractor similarity when one is searching for an object in a cluttered visual field appears to be exactly the opposite of its effect when one is identifying a single object whose location is known. One objective of the present study was to determine whether searching for an object off the masking plane would alter the manner in which the similarity of targets to maskers affects search behavior.

\section{The purposes of the present study}

One of the purposes of this study was to investigate the degree to which target search and comparison is affected by binocular disparity differences between the target objects (always presented in zero disparity) and the masking objects (presented with either zero, positive or negative disparities). Presenting the maskers with a non-zero disparity leads them to be perceived to be on a separate depth plane than that occupied by the targets. The question here is whether such disparity differences between maskers and target would affect visual search behavior. The experimental task required discriminating among targets (letters) simultaneously presented at four different locations on the fixation plane. Specifically, four symmetrically positioned target letters were presented stereoscopically on the fixation plane (zero disparity). In half of the trials, the four target letters were identical; in the other half of the trials, three were identical and the fourth was different. Participants were instructed to judge whether the four letters were the same or not. The first objective was to determine whether the introduction of a binocular disparity difference between the target plane (zero disparity) and the masker plane (positive or negative disparity) would affect performance when the masking plane contained recognizable objects (randomly rotated letters).

The other purpose of this study was to investigate whether the nature of the objects appearing on the masker plane affected the degree to which the direction of disparity differences between the masker and target planes affected performance. In this study, a letter masker and three types of control maskers were employed. The letter masker consisted of randomly selected and oriented letters of the alphabet uniformly spaced along the horizontal and vertical axes of the masker plane (Fig. 1a). The masker plane appeared either behind (uncrossed disparity), in front of (crossed disparity), or on the fixation plane (no disparity). If nearby objects are more likely to capture attention than more distant ones, we would expect better discrimination between the two types of target (all four letters the same versus three of them the same and one different) when the masking plane was perceived to be behind the target plane, rather than when the objects on the masking plane appeared to be either in front of or on the same plane as the target.

The second type of masker was constructed by taking the Fourier transform of a letter masker, randomizing its phase spectrum, and reconstructing a new image using the original amplitude spectrum of the letter masker but with phase randomized. Figure 1c presents the randomized phase masker constructed from the letter masker (Fig. 1a). Although the randomized phase masker has exactly the same amplitude spectrum as the letter masker, it appears more like a noise in that it lacks contours or objectlike characteristics. If the asymmetrical unmasking effect of the location of a letter masker (relative to that of the target) depends on the object-like characteristics of the letters in the masker, we would expect this asymmetry to disappear when the phase spectrum is randomized to remove the object-like characteristics of the letter masker. Hence we might expect to find equivalent amounts of unmasking with a random-phase masker, independent of whether the target appears in front of, or behind the masker.

The third type of masker consisted of the random rearrangement of the pixels of the letter masker (Fig. 1d). This preserves the root mean square (RMS) contrast of the letter masker but removes all object-like characteristics, and produces a flat amplitude spectrum (equal energy at all spatial frequencies). This masker retains the RMS contrast of the letter masker but destroys any object-like characteristics, and produces a uniform spatial frequency spectrum.

The fourth type of masker consisted of fragments of letters (Fig. 1b). These letter fragments had object-like characteristics (uniform luminance profile within sharp borders) but were not objects that could be labeled and were unlikely to elicit the same degree of lexical activity as a letter masker. If the asymmetrical masking effect is dependent on the object-like characteristics of the fragments on the masker plane, we would expect to also observe an asymmetrical masking effect with this kind of letter-fragment masker. If, on the other hand, the asymmetrical effect depended on the objects being recognizable, the asymmetrical effect might disappear. 


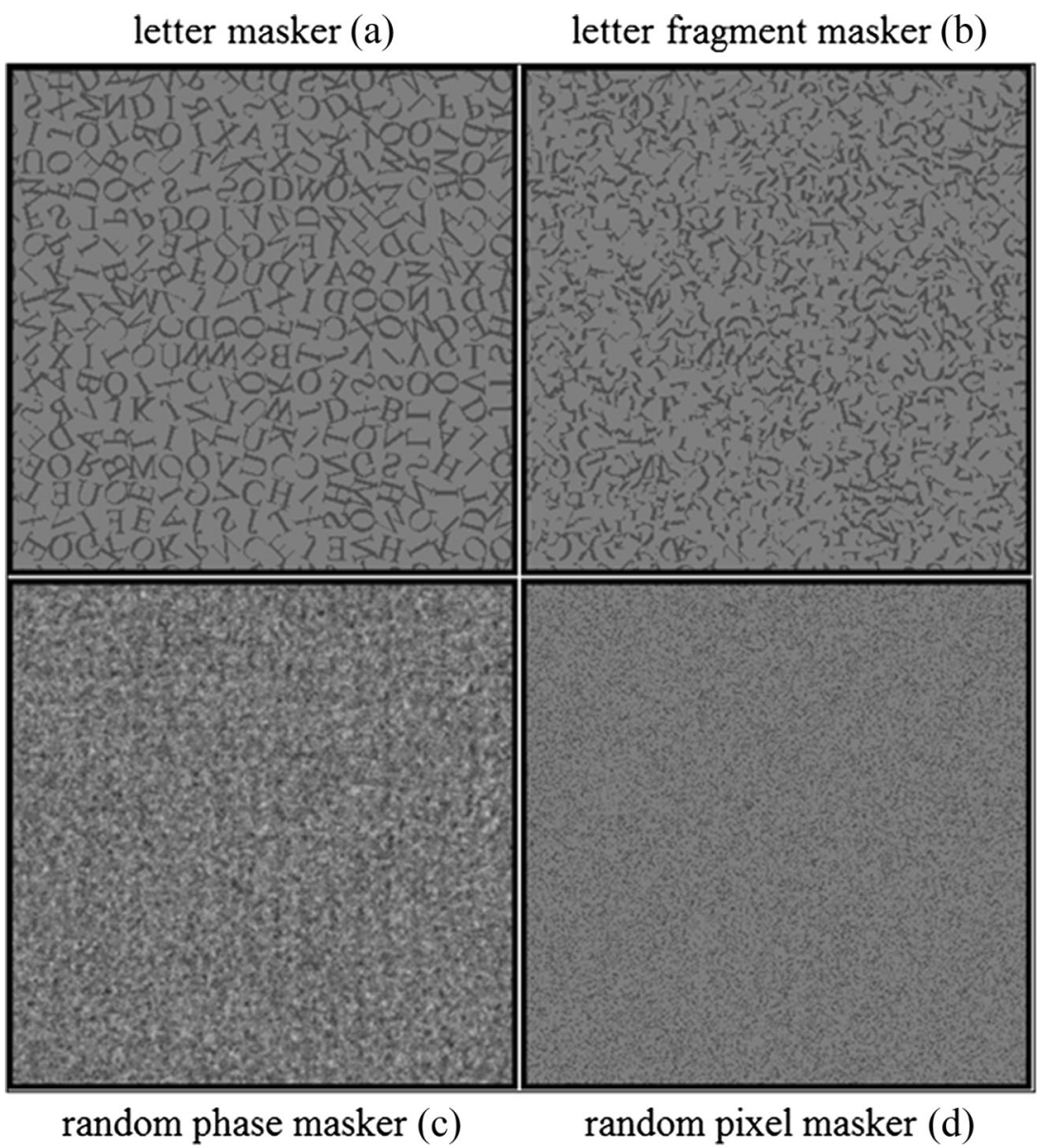

Fig. 1 Examples of the four maskers employed in this experiment

In this study, in addition to the disparity and type of masker, the third independent variable was the duration of the presentation of the target stimuli and maskers.

\section{Methods \\ Participants}

Twenty-four students studying at Peking University (10 males and 14 females) with ages between 17 and 26 years (mean age, 22.5 years) participated in the experiment. They were screened for normal or corrected-to-normal visual acuity (Tumbling E chart). The participants gave their written informed consent and were paid a modest stipend for their participation.

\section{Stimuli}

The stimuli were presented on a SyncMaster 788DF computer monitor with a spatial resolution of $1,024 \times 768$, a refresh rate of $80 \mathrm{~Hz}$, and a black background. Using a mirror stereoscope, stimuli displayed on the monitor were presented to participants who were seated with a distance of $45 \mathrm{~cm}$ from the monitor screen and head-restrained by a chin and forehead rest. The size of each of the two square half fields of the visual stimuli (i.e., the left and right fields for the stereoscopic presentation of stimuli) on the monitor screen was $13.3^{\circ} \times 13.3^{\circ}$ $(10.5 \mathrm{~cm} \times 10.5 \mathrm{~cm})$.

The binocularly presented target letters always appeared on the fixation plane. Surrounding the center fixation point of each of the two half fields, the target stimulus consisted of four symmetrically positioned English letters (font type = Times New Roman, font size $=24$ points, Fig. 3), whose positions were top, bottom, left, and right to the center fixation point, respectively. The separation between the two letters that were along the center horizontal line and the separation between the two letters that were along the center vertical line was $4.0^{\circ}$. For a single test trial, either all the four letters were the same (same-letter trials) or three letter were the same with one letter different (different-letter trials). The pixel luminance of a target letter was set to be 10 pixel-luminance units below that of the masker to which it was added. Table 1 presents the letters that could appear as targets. On same-letter trials, one of the target letters was randomly selected to appear in all four positions on the fixation plane. On different-letter trials, a 
Table 1 The 15 target letters used in the Experiment are listed. On 'same' trials, one of these 15 target letters was chosen at random and presented in the four cardinal positions. On 'different' trials, one of the target letters was chosen at random and presented at three of the four cardinal positions (randomly chosen). The letter placed in the fourth cardinal position on 'different' trials was the one paired with the target letter

\begin{tabular}{ll}
\hline Target letter & Different letter \\
\hline O & U \\
G & C \\
B & R \\
Q & D \\
T & F \\
X & K \\
A & H \\
W & M \\
U & V \\
V & Y \\
P & D \\
M & N \\
J & L \\
B & W \\
O & D \\
\hline
\end{tabular}

target letter was randomly selected and appeared in three of the positions (randomly chosen), a different letter of the pair appeared in the remaining position. Same-letter targets were presented on $50 \%$ of the trials.

Four types of binocular maskers were employed: letter masker, letter-fragment masker, phase masker, and pixel masker (see Fig. 1). The left- and right-eye letter maskers consisted of the same random selection of upper case letters arranged in an 18 by 18 grid, with the angle of orientation of each letter in the masker being randomized. The letters were added to a uniform gray field with a pixel luminance of 127 $\left(33 \mathrm{~cd} / \mathrm{m}^{2}\right)$. The pixel luminance within each letter was set to 77 pixel values darker than the gray field (the letter appeared to be darker than the background). Each masking field was surrounded by a 4-pixel-wide black border with a pixel luminance of $255\left(<0.1 \mathrm{~cd} / \mathrm{m}^{2}\right)$.

Random-phase maskers were constructed from the letter maskers by taking the Fourier transform of a letter masker, and reconstructing its image after randomizing its phase spectrum (Figs. 1 and 2).

A letter fragment masker was constructed from a letter masker by breaking each letter in the letter masker into four quadrants, and then randomly orienting the letters in each quadrant and then scrambling the position of the quadrants subject to the constraint that the randomization could not reproduce the original letter (Figs. 1 and 2).

The random pixel masker was constructed by randomly rearranging the pixels from a letter masker (Figs. 1 and 2). Hence the three maskers constructed from a letter masker
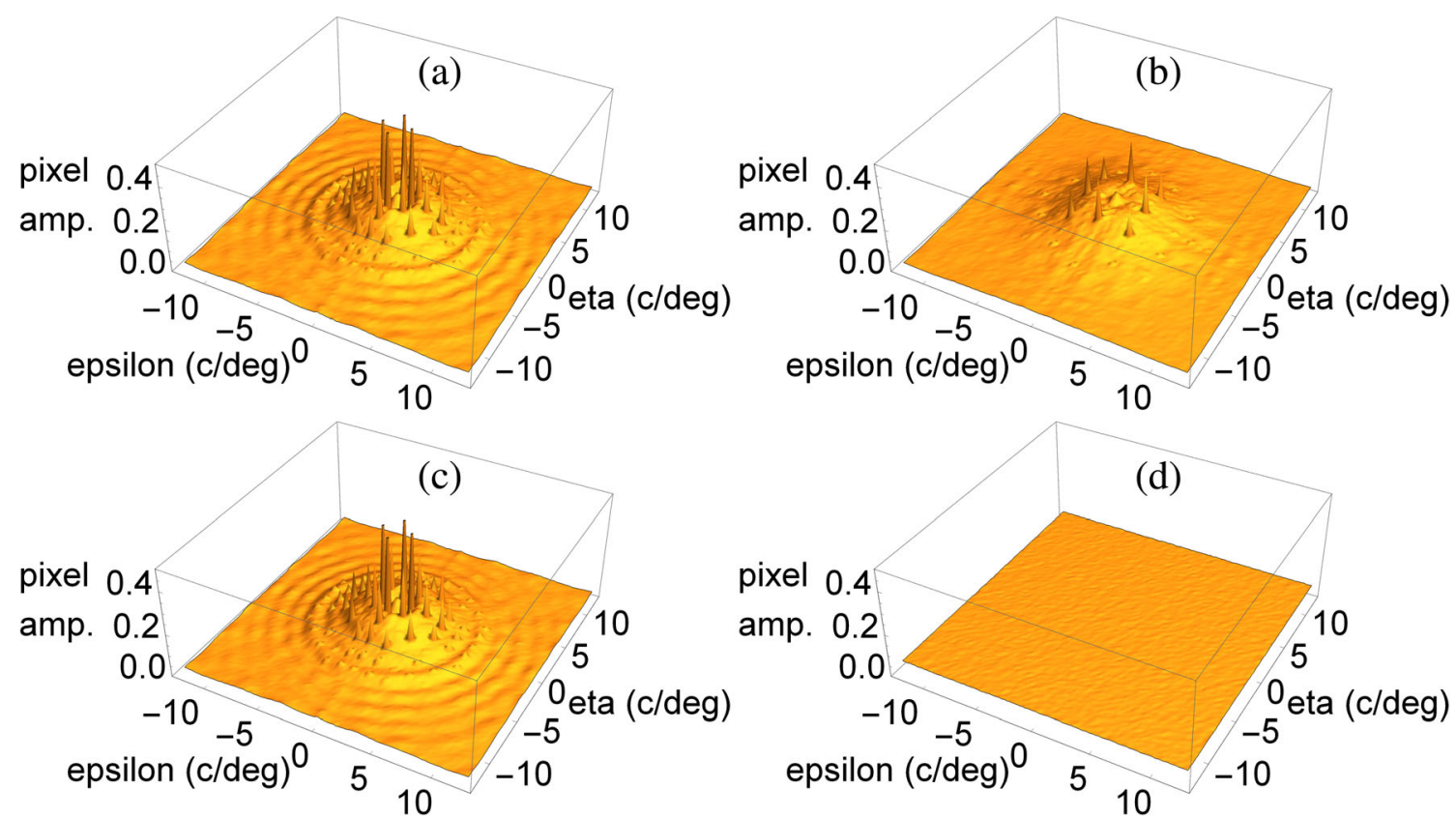

Fig. 2 Average two-dimensional amplitude spectra of the four different types of maskers. a Letter Masker. b Letter fragment masker. c Phase Masker. d Pixel Masker. (The average spectrum was computed over

100 different randomly generated maskers.) The DC component (the average pixel luminance) is not shown 


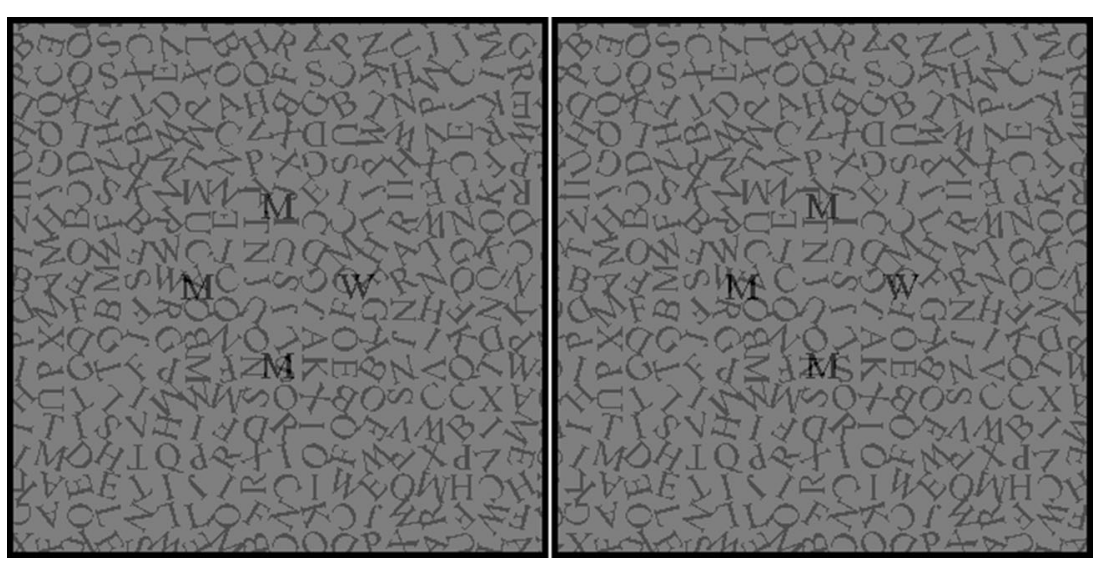

Fig. 3 A sample of the left- and right-eye views of the target stimuli superimposed on a letter-masker background. The targets occupy the same position within the frame in the left- and right-eye view and therefore are seen in zero screen disparity. The right-eye masker is shifted
7 pixels to right of its position in the left eye and therefore is seen in uncrossed screen disparity. Hence the masker is perceived as being on a plane that is behind the target plane had the same root mean square luminance as that of the letter masker.

Figure 2 plots the average two-dimensional amplitude spectrum of a letter masker (a), and the letter fragment masker (b), phase masker (c), and pixel masker (d) constructed from the letter masker. Note that the letter masker and phase masker have the same amplitude spectrum. The amplitude spectrum of the letter-fragment masker, although similar to that of the letter masker, is not identical, whereas the spectrum of the pixel masker is flat.

The fixation point was always presented precisely in the center of both the left- and right-eye screens, producing a screen disparity of zero for the fixation point. The four target stimuli also appeared in the same positions in both the leftand right-eye screens so that they also produced a screen disparity of zero. The maskers were presented alternatively with: (1) a screen disparity of 0 (the locations and luminances of the pixels in the left-eye frame were identical to this in the righteye frame); or (2) a crossed screen disparity of 7 pixels (16.67 arc min) such that the masker pixels in the left-eye frame were shifted 7 pixels to the left in the right-eye screen; or (3) an uncrossed screen disparity of 7 pixels such that the masker pixels in the left eye screen were shifted 7 pixels to the right in the right-eye screen. The left- and right-eye images were presented to the participant using a mirror stereoscope. Therefore, under the assumption that the fixation point was imaged on the fovea, the maskers could appear with either zero disparity (on the same plane as the target), crossed disparity (masker in front of the target), or uncrossed disparity (masker behind the target). Figure 3 shows an example in which the targets appeared with a screen disparity of zero (which was always the case for the targets), and the masker was presented in uncrossed disparity. When these images were presented to the left- and right-eyes, the viewer perceived the masker to be behind the target letters.

\section{Procedures}

The completely-crossed, within-subject design consisted of 4 Masker Types (letter, letter-fragment, phase, and pixel maskers); 3 Disparity Types (crossed, no disparity, uncrossed) and 4 Durations (100, 400, 700, 1,000 ms). Twenty trials were presented in each of the 48 combinations 4 Masker Types $\times 3$ Disparity Types $\times 4$ Durations with 10 trials assigned to the "same-letter" presentation (all the four letters were the same) and another 10 trials assigned to the "different-letter" presentation (one letter was different from other three letters, with the location of the letter that was different being randomly chosen). Trials were blocked across the 4 Durations and a Latin Square design was used to determine the order with which each participant encountered these 4 Duration blocks.

Within each Duration Block, there were 240 trials consisting of 20 trials at each combination of 4 Masker Types and 3 Disparity Types. These 240 trials were randomly ordered within each of the Duration Blocks. The participants were instructed to press a key to indicate whether the four letters of the target were the same (keypad 1) or different (keypad 2) as quickly as possible. No feedback was given.

As shown in Fig. 4, each test trial consisted of the sequential presentation of four displays. The trial started with a 500-ms presentation of a fixation cross at the center of the left- and right-eye displays. This was followed by the simultaneous presentation of the masker and target at one of the four durations. Following the termination of the target stimulus, a Gaussian noise image (mean pixel luminance $=127$ ) was presented for $100 \mathrm{~ms}$ with zero disparity. After the Gaussian noise mask, the monitor presented a black screen for the duration of the response period (2s). 
A short training session, consisting of three parts, was conducted to ensure that all participants were sensitive to the differences in disparity conditions, could accurately determine whether the four target letters were the same or different, and had become familiar with the task. The first part consisted of 12 trials, in which a participant was asked to indicate whether the target stimuli appeared to be either in front of, behind, or on the same plane as the masker. In these 12 trials the display remained on until the participant responded. A participant had to correctly identify the type of disparity on all 12 trials to continue in the experiment. This condition was included to ensure that none of the participants were stereo blind.

The first set of 12 trials was followed by a second set of 12 trials, in which the participant was asked to indicate whether the four targets were the same or different. Again the stimulus remained on until the subject responded. To continue in the experiment, the participant had to be correct on all of these 12 trials, to ensure that the participant could perform the task under all three disparity conditions, given an unlimited amount of time to peruse the visual display.

These two sets of 12 trials were followed by 24 trials with stimulus duration set to $100 \mathrm{~ms}$ to familiarize the participant with the main part of the experiment.

\section{Results}

Figure 5 plots average percent correct as a function of presentation duration for each of the three binocular disparities when the target was masked by one of four types of masker (letter, letter-fragment, phase, or pixel). A 4 Masker Type (letter masker, letter-fragment masker, phase masker, pixel masker) by 3 Disparity Type (crossed, uncrossed, none) by 4 Duration $(100,400,700,1,000 \mathrm{~ms})$ repeated-measures within-subject ANOVA found significant main effects of Masker Type $\left(\mathrm{F}_{2.333,53.650}=173.811, p<0.001\right.$, Greenhouse-Geisser correction), Masker Disparity $\left(\mathrm{F}_{2,46}=49.203, p<0.001\right)$, and Duration $\left(\mathrm{F}_{3,69}=58.348, p<0.001\right)$. In addition, the two-way interactions between Masker Type and Duration $\left(\mathrm{F}_{9,207}=\right.$ 7.544, $p<0.001)$, Masker Disparity and Duration $\left(\mathrm{F}_{4.116,94.658}=4.488, p=0.002\right.$, Greenhouse-Geisser correction), and Masker Type and Masker Disparity $\left(\mathrm{F}_{6,138}=3.719\right.$, $p=0.002$ ), were also significant. The three-way interaction between Masker Type, Masker Disparity, and Duration was also significant $\left(\mathrm{F}_{18,414}=2.824, p<0.001\right)$.

To better describe the nature of the three-way interaction, the following regression model was fit to the data.

$y_{i, j, k, m}=M D_{i, j}+b 1_{i, j} x_{k}+b 2_{i, j} x_{k}^{2}+e_{i, j, k, m}$

where $y_{i, j, k, m}$ is the percentage of correct responses obtained by subject $m(1 \leq m \leq 24)$ when the target and masker was presented with masker type $i(1 \leq i \leq 4)$ at disparity $j(1 \leq j \leq$ $3)$ with a duration equal to $x_{k}(1 \leq k \leq 4) . M D_{i, j}$ represents the average contribution of being in disparity condition $j$ when the target was masked by masker $i ; x_{k}(1 \leq k \leq 4)$ is the masker duration; $b 1_{i, j}$ and $b 2_{i, j}$ represent the linear and quadratic contributions of stimulus duration to performance when the participant is being tested in disparity condition $j$ when the target was masked by masker $i ; e_{i, j, k, m}$ is a random normal deviate.

In the "Appendix" we show that the number of parameters that are needed to fit the data can be reduced from the 48 parameters that are fit in a repeated-measures ANOVA, to 10 parameters in the regression analysis, without significantly affecting the goodness of fit of the model to the data. ${ }^{1}$ The solid lines in Fig. 5 represent the predictions of this model when the masking letters appear behind the target (uncrossed disparity), the dashed lines with the larger dashes represent the predictions when the masking plane appears in front of the target letters (crossed disparity), and the dashed lines with the smaller dashes represent the predictions when the target and masker appear on the same plane (no disparity). Several features of the model's predictions are worth noting. First, for letter maskers, there is no evidence that performance improves with duration when the masking letters appear either on the same plane as the target (no disparity) or in front of it (crossed disparity). ${ }^{2}$ Second, for phase maskers, there is no evidence that the functions relating performance to duration differ between the condition where the target letters and maskers were on the same plane (no disparity) versus the condition where the masking letters were perceived to be in front of the target letters (crossed disparity). ${ }^{3}$ Third, for letterfragment maskers, the function relating performance to duration is steeper when the masking plane is in front of the target plane (crossed disparity) that when the masking letters appear on the same plane as the target letters (no disparity). ${ }^{4}$ Fourth, for the pixel masker, although the functions relating performance to duration have the same shape, the function for the condition in which the target letters are on the same plane as the masker (no disparity)

\footnotetext{
${ }^{1}$ Any further attempts to reduce the number of parameters resulted in a significant increase in the error sum of squares associated with the reduced model relative to the full model.

${ }^{2}$ The null hypothesis that the functions relating performance to duration were both flat and identical for the zero disparity and crossed disparity conditions when a letter masker was employed could not be rejected. See "Appendix".

${ }^{3}$ The null hypothesis that the functions relating performance to duration were identical for the zero disparity and cross disparity conditions when a phase masker was employed could not be rejected. See "Appendix".

${ }^{4}$ The null hypothesis that the functions relating performance to duration were identical for the zero disparity and cross disparity conditions when a letterfragment masker was employed was rejected, but the null hypothesis that these two functions had different growth rates but the same intercept could not be rejected. See "Appendix".
} 
falls below that of the other two functions which do not appear to differ. ${ }^{5}$ Fifth, for the letter maskers, letterfragment maskers, and phase maskers, when the masking plane is behind the target plane (uncrossed disparity), the rate of growth in performance as a function of duration exceeds that of the other two disparity conditions. ${ }^{6}$

To examine the two-way interactions, we collapsed over duration and determined the average percent correct for the 12 combinations of Masker Type and Disparity Type. Figure 6 presents a plot of the collapsed data. The effect of disparity is indicated by the difference in percent correct for the crossed (masker in front of target) and uncrossed disparity (masker behind the target letters) when they are compared to the zero disparity conditions (target on the same plane as the masker). Figure 6 shows that uncrossed disparity (masker behind the target plane) produced a significant improvement in performance over the zero disparity condition for all four maskers, but that when the disparity was crossed (target behind the masker), improved performance (relative to the zero disparity condition) was only observed in the letter-fragment condition. When the target appeared in front of the masker background, the improvement in performance relative to the zero disparity condition did not differ significantly between the letter and letter fragment conditions ( $\mathrm{t}[23]=2.09, p>0.05$ ). In addition, when the target appeared in front of the background, the improvement in performance relative to the zero disparity condition did not differ between the unpatterned maskers (phase and pixel maskers). However, when the target appeared in front of the masker, the improvement in performance relative to the zero disparity condition was significantly better for the patterned maskers (letter maskers and letter-fragment maskers) than for the unpatterned maskers (phase maskers and pixel maskers) $(\mathrm{t}[23]=4.16, p<0.001)$. However, when the target appeared behind the masking plane, the improvement in performance relative to the zero disparity condition for the patterned maskers did not differ from those of the noise maskers $(\mathrm{t}[23]=0.90, p>0.25)$.

In general, average percent correct as a function of type of masker was ordered from lowest to highest as letter masker, letter-fragment masker, phase masker, and pixel masker (for all pairwise comparisons, $p<0.001$ ), with performance being best when the target was in front of the masker, followed by the target being behind the masker and the target on the same plane as the masker (for all pairwise comparisons, $p<0.001$ ).

\footnotetext{
$\overline{5}$ The null hypothesis that the functions relating performance to duration were identical for all three disparity conditions was rejected when a pixel masker was employed, but the null hypothesis that the shapes of the three functions were identical but that the intercept for the zero disparity condition differed from the other two could not be rejected. See "Appendix".

${ }^{6} \mathrm{~F}$-tests of the null hypothesis that the function relating performance to duration when the target letters appeared in front of the masker were the same as those for the other two disparity types were rejected for letter maskers, letterfragment maskers, and phase maskers. See "Appendix".
}

\section{Discussion}

\section{Are target comparisons easier when the target plane appears in front of the masker plane?}

The plane on which the target stimuli appeared was randomized from trial to trial in the present experiment to determine whether the ability to search for and compare targets was differentially affected by the location of the masker plane relative to that of the target plane (in front of, on the same plane, or behind the target plane). Figure 5 shows that, in the zero disparity condition, performance improved with stimulus duration in three of the four masker conditions. Because the views presented to the two eyes in the zero disparity condition are identical, this condition is equivalent to monocular viewing where there are no stereo depth cues that could enhance performance. The purpose of the experiment was to determine the extent to which the introduction of stereo cues that positioned the masking letters either behind or in front of the target letters would improve performance. Figure 5 clearly indicates the search advantage of having the masker appear to be behind the target letters (uncrossed disparity), rather than on the same plane or in front of the target letters (crossed disparity), was present for letter maskers, randomized phase maskers, and letter fragment maskers. Of particular interest is the fact that this advantage was maintained even when the spectral composition of a noise masker was identical to that of the letter masker. Hence the search advantage provided by having the masking letters appear behind the target letters does not depend on whether the masker plane consists of visual noise or of recognizable objects (letters) or even relatively non-recognizable shapes (letter fragments).

Since the nature of the masking plane (whether it consists of recognizable shapes or random noise) is not responsible for the advantage gained by having the masker plane appear to be behind the target plane, what then is responsible for this substantial advantage? Previous studies have indicated that there is no such advantage for the detection of a simple Gabor target presented against a noise background. From an evolutionary viewpoint, however, we might expect nearby objects to have greater salience than more distant ones (Franconeri and Simons, 2003). Hence when there is uncertainty as to the location of the target objects in depth (as there was in this experiment), we might expect those objects that are nearest to the observer to be processed first. When the target letters appear in front of the letters on the masking plane, we might expect search and comparison operations to be more rapid than when the searched-for objects appear to be behind the masking plane. The present data are consistent with such a hypothesis. 
Does the object nature of the masking plane affect the ease with which targets can be compared?

The second objective was to determine whether the presence of recognizable objects on the masking plane affected target search. A comparison of the letter masker and either the letter fragment or random phase maskers (see Fig. 5) indicates that the degree of masking under all three disparity types is more severe when the masking plane consists of recognizable objects (letters) than when it consists of either letter fragments or spectrally identical visual noise. It is also less likely that the letter fragment and random phase maskers would elicit significant activity in the neural networks leading to object formation and lexical activity than would letter maskers. Hence we might expect the letter masker to interfere more with the processing of the target letters than the other types of maskers. ${ }^{7}$ This conjecture is supported by the fact that an increase in the number of non-target letters in a search reduces the size of the congruency effect ${ }^{8}$ to a greater extent when the non-target letters are presented in an upright position (and could possibly initiate competing lexical activity) than when they are inverted (and presumably less likely to initiate lexical activity; see Chen \& Cave, 2013).

To evaluate the potential role of lexical interference, we subtracted each participant's percent correct for the letter masker from his or her percent correct for the phase masker for each of the three disparity conditions. A 3 Disparity by 4 Duration repeated measures ANOVA of the difference scores (Phase Masker - Letter Masker) revealed a significant Main Effect of Duration $(\mathrm{F}[3,138]=12.112, p<0.001)$ but no effect of Disparity Type or Disparity Type by Duration interaction. The fact that the linear portion of the Duration effect was both positive and highly significant $(\mathrm{F}[1,23]=34.507, p<0.001)$ indicates that performance increased more rapidly with duration for a phase masker than for a letter masker for all three disparity types. Hence, when the task involves a visual search of a plane for a match or mismatch of letter types, the greater the similarity between the objects on target plane and the

\footnotetext{
${ }^{7}$ In hearing, peripheral or energetic masking is said to occur when the target and masker simultaneously activate the same regions on the cochlear partition. Any interference caused by the masker at either central auditory or cognitive levels of processes is referred to as informational masking (Durlach et al., 2003, Freyman, Helfer, McCall, \& Clifton, 1999; Schneider, Li, \& Daneman, 2007). In vision, any interference that could be attributed to an overlap between the spatial frequency characteristics of the target and masker would be analogous to energetic masking in audition. Interference at more central levels of visual processing (e.g., contour and object formation, lexical interference) would be analogous to informational masking in hearing. One could then interpret poorer performance on the letter discrimination task in the presence of a letter masker than in the presence of a fragmented letter masker as indicative of informational masking at a lexical level.

${ }^{8}$ The congruency effect refers to the fact that a distractor that appears at the fixation point has less of an effect on target search when it is the same letter as a peripherally presented target than when it is a different letter than the peripherally presented target.
}

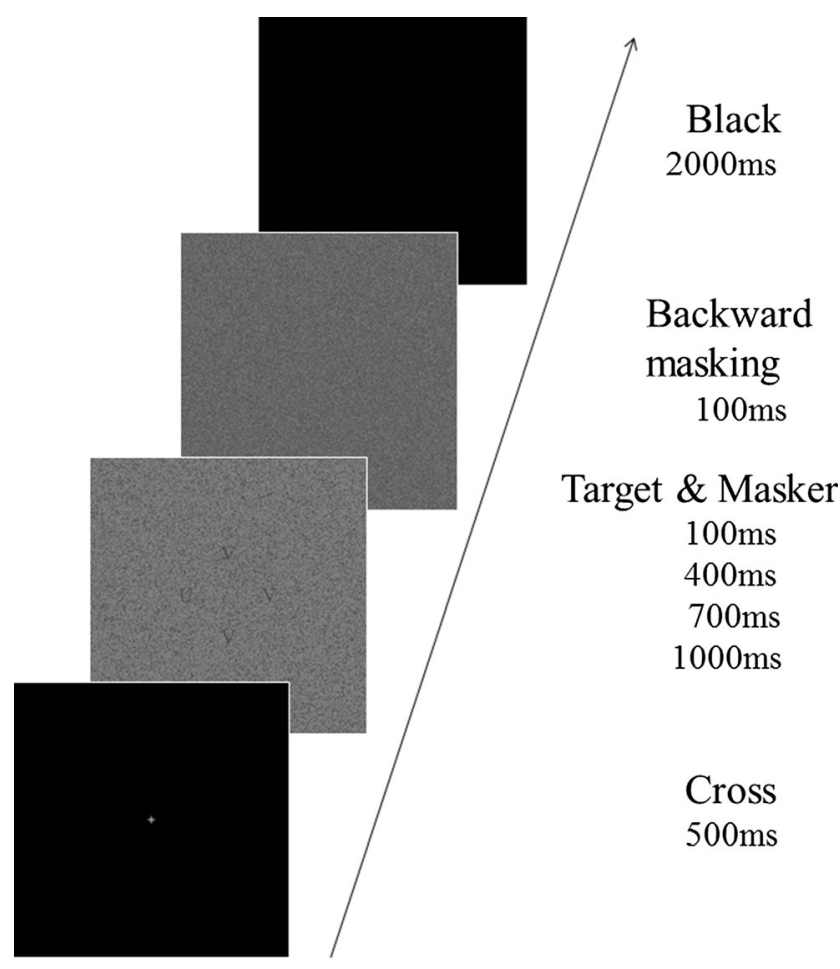

Fig. 4 Schematic diagram showing the sequence of events on a trial with the direction of the arrow indicating the sequence of events. After the presentation of a 500-ms cross, a binocularly presented target stimulus and masker was presented with the stimulus-presentation duration of either $100,400,700$, or 1,000 ms, followed by a 100-ms random dot masker, with the screen blackened during the response period

masker plane, the greater the degree of masking, even when the spatial frequency composition of the two types of maskers is identical.

It is also the case that the rate of improvement in performance with duration is more rapid for the letter-fragment masker than for the letter masker. A 3 Disparity by 4 Duration repeated measures ANOVA of the difference scores (LetterFragment Masker - Letter Masker) revealed a significant Main Effect of Duration $(\mathrm{F}[3,138]=7.174, p<0.001)$ but no effect of Disparity Type or Disparity Type by Duration interaction. Again, the linear portion of the Duration effect was both positive and highly significant $(\mathrm{F}[1,23]=16.749, p<0.001)$, indicating that performance increased more rapidly with duration for a letter-fragment masker than for a letter masker for all three disparity types. Given the similarity (but not identity) of the two-dimensional amplitude spectra of the two types of maskers, it is likely that the greater improvement in performance with duration for the letter-fragment masker than for the letter masker suggests that the letter masker may be interfering with the search process at an orthographic level. A search of the target plane requires the participant to determine whether all four letters are the same or one of them is different. Identification of whether or not the letters are identical is likely to require activation of the orthographic system. The letters in the letter masker are also likely to activate orthographic 


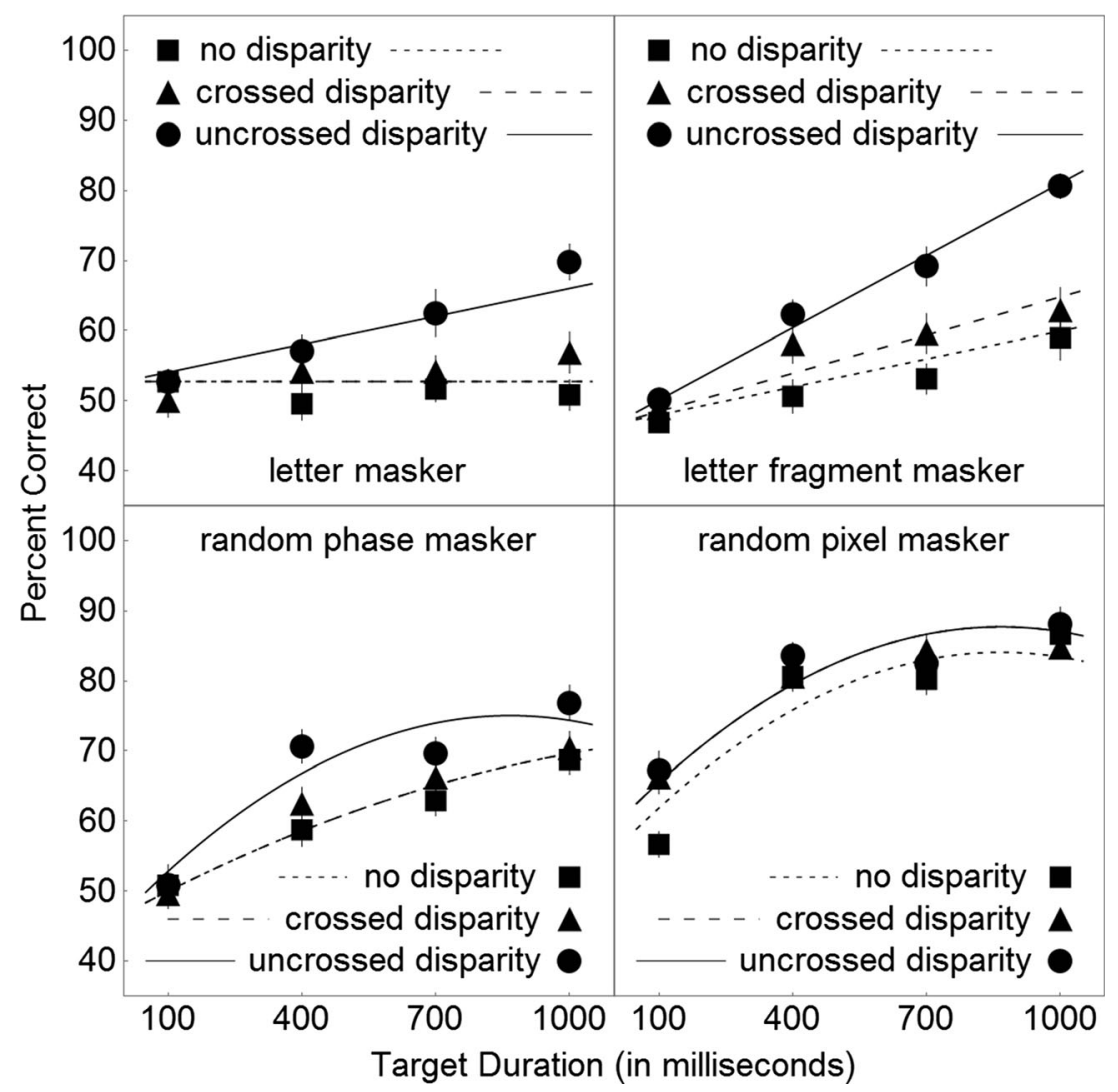

Fig. 5 Average percent correct as a function of stimulus duration for the four types of maskers and three types of disparity. Chance level performance is $50 \%$ correct. Standard error bars are shown

representations that could interfere with the search and comparison process leading to poorer performance for a letter masker than for a letter fragment masker. Hence this experiment provides evidence that when a masking background is likely to initiate activity in the systems that are employed in performing the search task, task performance will be adversely affected.

Interestingly, when the task requires identification of a target letter at a known location, letter identification accuracy is better for a letter masker than it is for a letter-fragment masker, and worst of all for a phase masker (Rabaglia \& Schneider, $2016^{9}$ ) - the exact opposite of what was found in the search and match task used in the present study. Rabaglia and Schneider suggested that when the target location is known, it is easier to maintain focused attention on the target location when the surrounding visual field consists of a collection of recognizable objects (e.g., letters), than when it consists of objects whose shapes are not easily identifiable (e.g., letter fragments). Previous studies (Auckland, Cave, \& Donnelly, 2007; Davenport \& Porter, 2004) have shown that object recognition is faster when the target object "fits" into the background within which it appears (e.g., a football player in a stadium) than when

\footnotetext{
$\overline{9}$ In the Rabaglia and Schneider study, both masker and target were presented on the same plane.
}

it is presented in an incongruent background (e.g., a football player on an opera stage). In addition, they argue that when the spatial frequency pattern on the retina can be resolved into a collection of forms (letters or letter fragments), that the resultant formation of the surrounding objects unmasks the target letter. When, however, the task requires a search and compare strategy, the greater the degree of similarity of the objects in the masking plane to the four target objects, the greater the degree to which it interferes with the neural mechanisms involved in performing this task. The results of Rabaglia and Schneider, along with those of the present study, indicate that degree of similarity between the target objects and the objects in the background have opposite effects when search is required than when the exact location of a target is known.

Finally, the degree of masking is more closely related to the degree of spectral overlap between the targets and the maskers than it is related to the RMS contrast of the masker. Performance was best for the pixel masker which had the same RMS contrast as the other maskers but whose spectral composition was flat rather than concentrated at the spatial frequencies represented by the target. Moreover, the rate of growth in performance as a function of duration was much more rapid for this masker than for the other maskers and did not differ much with respect to the disparity condition. This indicates that the advantage of having the target appear in front of the masker in 


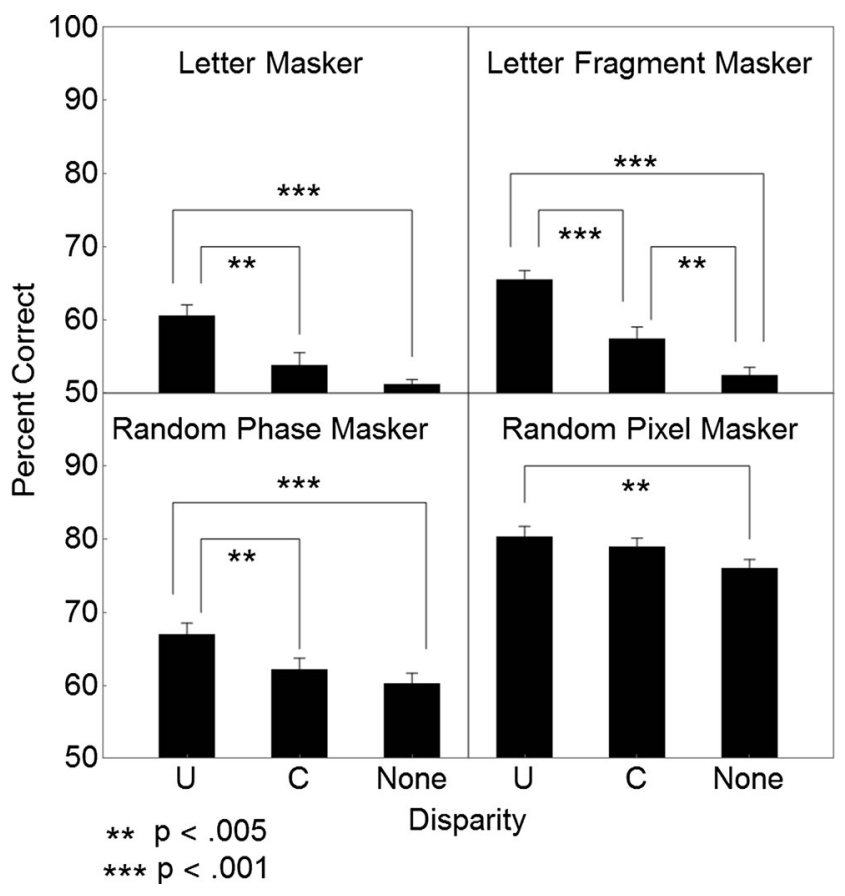

Fig. 6 Average percent correct at each of the three disparities for the four different types of maskers. Chance level performance is $50 \%$ correct. Standard error bars are shown

this task is primarily restricted to those cases in which there is a close degree of overlap between the spatial composition of the target letters and the spatial composition of the masker.

\section{Limitations}

In the present study we found that performance on a search and comparison task was more accurate when the letter masker was presented in uncrossed disparity (appeared behind the target plane) than when it appeared on the same plane or in crossed disparity (appeared in front of the target), whereas the sign of the disparity of the masker had no effect on the detection of a Gabor stimulus in noise. It is possible that the type of task (detection vs. search and comparison) is responsible for the differential effect that the type of disparity has on performance. Alternatively, it is possible that the differential effect is due to the nature of the target stimuli employed in these stereoscopic tasks (Gabor vs. letters). We are now investigating, when the target is presented in a fixed and known location, the extent to which stereoscopic cues can affect identification of a single target letter. If the asymmetrical effect of disparity is due to the type of task, it might not be as apparent in the identification task.

The present study also found that performance was much poorer for letter maskers than for letter fragment maskers and random phase maskers. We noted that this was consistent with the notion that letter maskers were more likely to initiate lexical activity than letter fragment maskers, which could account for the poorer performance when letter maskers were employed.
However, it is also possible that differences between the letters and letter fragments that are processed before the lexical access stage (such as the size and shapes of the individual masker elements) could be contributing to this effect. Hence the relative contribution of differential lexical interference versus lowerorder size and shape differences to the performance differences among maskers remains to be determined.

\section{Summary}

1. The degree of binocular unmasking due to disparity in a visual search and comparison task is greater when the masking objects appear to be behind, rather than in front of or on the same plane as the target objects. These results contrast with those that indicate that when simple targets (e.g., Gabor patterns) are presented foveally (at the point of fixation) their detectability appears to be independent of whether they are perceived in front of or behind a noise masker.

2. In search tasks, the greater the similarity between the target objects and the masker, the greater the degree of masking, independent of whether the masker appears in front of, behind, or on the same plane as the targets. Letter maskers and letter-fragment maskers interfere with target search and comparison more than does a noise plane that is spectrally identical to the letter maskers.

3. The greatest degree of interference with the search and comparison task occurs for letter maskers. To determine whether one of the four target letters differs from the other three most likely involves higher-order lexical processes (identifying each of the letters before comparing them). The simultaneous engagement of these lexical processes by the letter masker is likely to interfere with the lexical identification and comparison of the target letters. This interference at the lexical level may explain why the letter masker interferes with the task more than the other maskers, independent of the relative positions of the target and masking planes.

4. The differential advantage conferred by having the target plane appear to be in front of the masker is limited to those conditions where there is a substantial degree of spectral overlap between target and masker.

5. The degree of similarity between the background and the target object has the opposite effect when the task requires identification of an object in a known location (no searching), suggesting that it is easier to keep attention focused on the target when the background consists of recognizable objects of the same type as the target. When the surround cannot be easily organized into recognizable objects, attention may be drawn away from processing the target, leading to poorer performance in an identification task. 
Acknowledgements This work was supported the "973" National Basic Research Program of China (2015CB351800), the Beijing Municipal Science \& Tech Commission (Z161100002616017), the National High Technology Research and Development Program of China (863 Program: 2015AA016306), "985" grants from Peking University, and the Natural Sciences and Engineering Research Council of Canada (RGPIN-9952-13). We thank Lan Wei for producing masking stimuli used in this study.

\section{Appendix}

The total error sum of the squares for the 4 Masker Types by 3 Disparity Types by 4 Durations reported in the "Results" section was 144,518 with 1,104 degrees of freedom. The total sum of squares for the linear regression model specified by Eq. 1 was 147,996 with 1,116 degrees of freedom. Hence the two models (repeated measures ANOVA and Linear Regression) provide equivalently good fits. We then were able to reduce the number of parameters in the Linear Regression Model to 10 without significantly affecting the error sum of squares. Specifically, we constructed the following composite null hypothesis to test whether the 10parameter model was as good as the 36 parameter model specified by Eq. 1 by assuming under this composite null hypothesis that some of the parameters were equal to one another, and others were zero. In the Linear Regression Model, the first subscript, $i$, represents the Masker Type with $i=1$ specifying the letter masker, $i=2$ specifying the letter fragment masker, $i=3$ specifying the phase masker, and $i=4$ specifying the pixel masker. The second subscript, $j$, represents the disparity of the masker with $j=1$ specifying the condition (uncrossed disparity) in which the masker appears behind the target, $j=2$ specifying the condition (zero disparity) in which the target letters appear on the same plane as the masker, and $j=3$ specifying the condition (crossed disparity in which the masker plane appears in front of the target letters. The third subscript, $k$, represents the duration of the trial with $k=1$ indicating a $100-\mathrm{ms}$ trial duration, $k=2$ indicating a 400-ms trial duration, $k=3$ indicating a 700-ms duration, and $k=4$ indicating a $1,000-\mathrm{ms}$ duration. Specifically, we tested the composite null hypothesis that

$$
\begin{aligned}
& H 0: M D_{1,1}=M D_{1,2}=M D_{1,3} \\
& M D_{2,1}=M D_{2,2}=M D_{2,3}=M D_{3,1}=M D_{3,2}=M D_{3,3} \\
& M D_{4,1}=M D_{4,3} \\
& b 1_{1,1}=b 1_{2,2} \\
& b 1_{1,2}=b 1_{1,3}=b 2_{1,1}=b 2_{1,2}=b 2_{1,3}=b 2_{2,1}=b 2_{2,2}=b 2_{2,3}=0 \\
& b 1_{2,1}=b 1_{3,2}=b 1_{3,3} \\
& b 1_{3,1}=b 1_{4,1}=b 1_{4,2}=b 1_{4,3} \\
& b 2_{3,1}=b 2_{4,1}=b 2_{4,2}=b 2_{4,3} \\
& b 2_{3,2}=b 2_{3,3}
\end{aligned}
$$

and failed to reject it $(\mathrm{F}[26,1116]=0.99, p=0.478)$. Hence a 10 -parameter regression model provides as good a fit as a 36 parameter regression model. The lines in Fig. 5 are the predictions of this 10-parameter model. The non-zero parameters of this model are:

$$
\begin{aligned}
& M D_{1,1}=M D_{1,2}=M D_{1,3}=52.74 \\
& M D_{2,1}=M D_{2,2}=M D_{2,3}=M D_{3,1}=M D_{3,2}=M D_{3,3}=46.65 \\
& M D_{4,1}=M D_{4,3}=59.34, M D_{4,2}=55.70 \\
& b 1_{1,1}=b 1_{2,2}=.0132719, b 1_{2,3}=0.0181527 \\
& b 1_{2,1}=b 1_{3,2}=b 1_{3,3}=0.0344126 \\
& b 1_{3,1}=b 1_{4,1}=b 1_{4,2}=b 1_{4,3}=0.0655073 \\
& b 2_{3,1}=b 2_{4,1}=b 2_{4,2}=b 2_{4,3}=-0.000037793 \\
& b 2_{3,2}=b 2_{3,3}=-0.0000114286
\end{aligned}
$$

To support the statements in footnote 2, we tested the null hypothesis that the functions relating performance to duration were identical and flat for the zero disparity and cross disparity conditions when a letter masker was employed. Specifically, we tested

$$
\begin{aligned}
H 0: & M D_{1,2}=M D_{1,3} \\
& b 1_{1,2}=b 1_{1,3}=b 2_{1,2}=b 2_{2,2}=0
\end{aligned}
$$

and failed to reject this null hypothesis $(\mathrm{F}[5,1116]=1.350, p=$ $0.241)$.

To support the statements in footnote 3 , we tested the null hypothesis that the functions relating performance to duration were identical for the zero disparity and cross disparity conditions when a phase masker was employed. Specifically, we tested

$$
\begin{gathered}
H 0: M D_{3,2}=M D_{3,3} \\
b 1_{3,2}=b 1_{3,3} \\
b 2_{3,2}=b 2_{3,3}
\end{gathered}
$$

and failed to reject this null hypothesis $(\mathrm{F}[3,1116]=1.145, p=$ 0.459).

To support the statements in footnote 4 , we tested the null hypothesis that the functions relating performance to duration were identical for the zero disparity and cross disparity conditions when a letter-fragment masker was employed. Specifically, we tested

$$
\begin{gathered}
H 0: M D_{2,2}=M D_{2,3} \\
b 1_{2,2}=b 1_{2,3}
\end{gathered}
$$

and rejected this null hypothesis $(\mathrm{F}[3,1116]=3.521, p=$ $0.015)$. We then tested the null hypothesis that permitted the rate of growth but not the intercept to differ between the zero disparity and cross disparity conditions when a letter-fragment masker was employed. Specifically, we tested

$H 0: M D_{2,2}=M D_{2,3}$

and failed to reject this null hypothesis $(\mathrm{F}[1,1116]=0.000, p=$ 0.997). Hence we concluded that the rate of growth differed between the zero and cross disparity conditions when a letterfragment masker was employed. 
To support the statements in footnote 5 , we tested the null hypothesis that the functions relating performance to duration were identical for all three disparity conditions when a pixel masker was employed. Specifically, we tested the null hypothesis that

$$
\begin{gathered}
H 0: M D_{4,1}=M D_{4,2}=M D_{4,3} \\
b 1_{4,1}=b 1_{4,2}=b 1_{4,3} \\
\quad b 2_{4,1}=b 2_{4,2}=b 2_{4,3}
\end{gathered}
$$

and rejected it $(\mathrm{F}[2,1116]=2.217, p=0.039)$. We then tested the null hypothesis that the shapes of the three functions were identical but that the intercept for the zero disparity condition differed from the other two. Specifically, we tested the null hypothesis that

$$
\begin{array}{r}
H 0: M D_{4,1}=M D_{4,3} \\
b 1_{4,1}=b 1_{4,2}=b 1_{4,3} \\
b 2_{4,1}=b 2_{4,2}=b 2_{4,3}
\end{array}
$$

and failed to reject it $(\mathrm{F}[5,1116]=1.377, p=0.230)$.

To support the statements in footnote 6 , we tested the null hypothesis that the functions relating performance to duration when the target letters appeared in front of the masker were the same as those for the other two disparity types for letter maskers, letter-fragment maskers, and phase maskers. The first null hypothesis was that the functions were all the same when a letter masker was employed. Specifically, we tested the null hypothesis that

$$
\begin{gathered}
H 0: M D_{1,1}=M D_{1,2}=M D_{1,3} \\
b 1_{1,1}=b 1_{1,2}=b 1_{1,3} \\
b 2_{1,1}=b 2_{1,2}=b 2_{1,3}
\end{gathered}
$$

and rejected it $(\mathrm{F}[2,1116]=8.437, p<0.001)$. The second null hypothesis was that the functions were all the same when a letter-fragment masker was employed. Specifically we tested the null hypothesis that

$$
\begin{gathered}
H 0: M D_{2,1}=M D_{2,2}=M D_{2,3} \\
b 1_{2,1}=b 1_{2,2}=b 1_{2,3} \\
b 2_{2,1}=b 2_{2,2}=b 2_{2,3}
\end{gathered}
$$

and rejected it $(\mathrm{F}[2,1116]=14.214, p<0.001)$. The third null hypothesis was that the functions were all the same when a phase masker was employed. Specifically we tested the null hypothesis that

$$
\begin{gathered}
H 0: M D_{3,1}=M D_{3,2}=M D_{3,3} \\
b 1_{3,1}=b 1_{3,2}=b 1_{3,3} \\
b 2_{3,1}=b 2_{3,2}=b 2_{3,3}
\end{gathered}
$$

and rejected it $(\mathrm{F}[6,1116]=3.551, p=0.002)$. Hence we concluded that the function for uncrossed disparity differed from the other two when either a letter masker, letterfragment masker or phase masker was employed.

\section{References}

Auckland, M. E., Cave, K. R., \& Donnelly, N. (2007). Nontarget objects can influence perceptual processes during object recognition. Psychonomic Bulletin \& Review, 14, 332-337.

Chen, Z., \& Cave, K.R. (2013). Perceptual load vs. dilution: the roles of attentional focus, stimulus category, and target predictability. Frontiers in Psychology: Cognition. doi:10.3389/fpsyg.2013.00327

Davenport, J. L., \& Potter, M. C. (2004). Scene consistency in object and background perception. Psychological Science, 15, 559-564.

de la Rosa, S., Moraglia, G., \& Schneider, B. A. (2008). The magnitude of binocular disparity modulates search time for targets defined by a conjunction of depth and color. Canadian Journal of Experimental Psychology, 62, 150-155.

Durlach, N. I., Mason, C. R., Kidd, G., Jr., Arbogast, T. L., Colburn, H. S., \& Shinn-Cunningham, B. (2003). Note on informational masking. Journal of the Acoustical Society of America, 113, 2984 2987.

Enns, J. T., \& Di Lollo, V. (2000). What's new in visual masking? Trends in Cognitive Sciences, 4, 345-352.

Fox, R., \& Patterson, R. (1981). Depth separation and lateral interference. Perception and Psychophysics, 30, 513-520.

Franconeri, S. L., \& Simons, D. J. (2003). Moving and looming stimuli capture attention. Perception \& Psychophysics, 65, 999-1010.

Freyman, R. L., Helfer, K. S., McCall, D. D., \& Clifton, R. K. (1999). The role of perceived spatial separation in the unmasking of speech. Journal of the Acoustical Society of America, 106, 3578-3588.

Heesy, C. P. (2009). Seeing in stereo: The ecology and evolution of primate binocular vision and stereopsis. Evolutionary Anthropology, 18, 21-35.

Howard, I. P., \& Rogers, B. J. (2012). Perceiving in Depth: 3-Volume Set. New York: Oxford University Press.

Howard, I. P., \& Rogers, B. J. (2002). Seeing in depth (Vol. 2: depth perception). Toronto: Canada University of Toronto Press.

Howard, I. P., \& Rogers, B. J. (1995). Binocular vision and stereopsis. New York: Oxford University Press.

Henning, G. B., \& Hertz, G. B. (1973). Binocular masking level differences in sinusoidal grating detection. Vision Research, 13, 24552463.

Lehmkuhle, S., \& Fox, R. (1980). Effect of depth separation on metacontrast masking. Journal of Experimental Psychology: Human Perception and Performance, 6, 605-621.

Manning, M. L., Finlay, D. C., Neill, R. A., \& Frost, B. G. (1987). Detection threshold differences to crossed and uncrossed disparities. Vision Research, 27, 1683-1686.

Moraglia, G., \& Schneider, B. A. (1990). Effects of direction and magnitude of horizontal disparities on binocular unmasking. Perception, 19, 581-593.

Moraglia, G., \& Schneider, B. A. (1992). On binocular unmasking of signals in noise: Further tests of the summation hypothesis. Vision Research, 32, 375-385.

Mustillo, P. (1985). Binocucar mechanisms mediating crossed and uncrossed stereopsis. Psychological Bulletin, 97, 187-201.

Nakayama, K., \& Silverman, G. H. (1986). Serial and parallel processing of visual feature conjunctions. Nature (London), 320, 264-265.

O’Toole, A. J., \& Walker, C. L. (1997). On the preattentive accessibility of stereoscopic disparity: Evidence from visual search. Perception and Psychophysics, 59(2), 202-218.

Otto, J. M., Bach, M., \& Kommerell, G. (2010). Advantage of binocularity in the presence of external visual noise. Graefes Archive for Clinical and Experimental Ophthalmology, 248, 535-541.

Patterson, R., Cayko, R., Short, G. L., Flanagan, R., Moe, L., Taylor, E., \& Day, P. (1995). Temporal integration differences between crossed and uncrossed stereoscopic mechanisms. Perception and Psychophysics, 57, 891-897. 
Pelli, D. G., \& Tillman, K. A. (2008). The uncrowded window of object recognition. Nature Neuroscience, 11, 1129-1135.

Rabaglia, C. D., \& Schneider, B. A. (2016). Age-related inhibitory deficit, or lack of familiarity benefit? Evidence from letter identification among visual distractors. Attention, Perception, \& Psychophysics, $78,542-565$.

Richards, W. (1970). Stereopsis and stereoblindness. Experimental Brain Research, 10, 380-388.

Richards, W. (1971). Anomalous stereoscopic depth perception. Journal of the Optical Society of America, 61, 410-414.

Schneider, B., Moraglia, G., \& Jepson, A. (1989). Binocular unmasking: An analog to binaural unmasking? Science, 243, 1479-1481.

Schneider, B. A., Li, L., \& Daneman, M. (2007). How noise interferes with speech comprehension in everyday listening situations? Journal of the American Academy of Audiology, 18, 559-572.
Speranza, F., Moraglia, G., \& Schneider, B. A. (1995). Age-related changes in binocular vision: detection of noise-masked targets in young and old observers. Journal of Gerontology: Psychological Sciences, 50B, 114-123.

Speranza, F., Moraglia, G., \& Schneider, B. A. (2001). Binocular detection of masked patterns in young and old observers. Psychology and Aging, 16, 281-292.

Treisman, A. M., \& Gelade, G. (1980). A feature-integration theory of attention. Cognitive Psychology, 12, 97-136.

Uttal, W. R., Fitzgerald, J., \& Eskin, T. E. (1975). Paramters of tachistoscopic stereopsis. Vision Research, 15, 705-712.

Wardle, S. G., Cass, J., Brooks, K. R., \& Alais, D. (2010). Breaking camouflage: Binocular disparity reduces contrast masking in natural images. Journal of Vision, 10(38), 1-12. 